\title{
VIBRATIONAL AND ROTATIONAL COOLING OF ELECTRONS BY MOLECULAR HYDROGEN
}

\author{
J. H. WAITE, JR.* and T. E. CRAVENS \\ Space Physics Research Laboratory, Department of Atmospheric and Oceanic Science, The \\ University of Michigan, Ann Arbor, MI 48109, U.S.A.
}

(Received 19 May 1981)

\begin{abstract}
The cooling of electrons by vibrational and rotational excitation of molecular hydrogen plays an important role in the thermal balance of electrons in atmospheres containing significant amounts of $\mathrm{H}_{2}$. Calculations of vibrational and rotational cooling rates of electrons by $\mathrm{H}_{2}$ are described. Results are presented for a wide range of electron and neutral temperatures. Analytical formulae for some of the cooling rates are also provided.
\end{abstract}

\section{INTRODUCTION}

Theoretical calculations of electron temperatures in the Jovian ionosphere were carried out by Henry and McElroy (1969), Prasad and Capone (1971), and Cravens (1974), assuming local energy balance, and by Nagy et al. (1976) who included the effects of thermal conduction. All these calculations employed neutral atmosphere models with low neutral temperatures (less than $300 \mathrm{~K}$ ), except for Nagy et al. (1976) who used neutral models with both low and high (greater than $1000 \mathrm{~K}$ ) neutral temperatures. All these calculations adopted the cooling rates presented by Henry and McElroy (1969) for the cooling of electrons by the vibrational and rotational excitation of molecular hydrogen. These cooling rates were presented only for neutral temperatures less than $300 \mathrm{~K}$.

The thermospheres of Jupiter and Saturn are now thought to have exospheric neutral temperatures which are greater than about $1000 \mathrm{~K}$ (Fjeldbo et al., 1976; Atreya et al, 1979; Kliore et al., 1980). Future theoretical calculations of electron temperatures for these planets, or any atmosphere containing $\mathrm{H}_{2}$, should use cooling rates which are appropriate for neutral temperatures greater than $1000 \mathrm{~K}$. The Ph.D. thesis of Waite (1980) presents a theoretical model of the ionosphere of Saturn (including electron and ion temperature calculations), as well as the results of calculations of $\mathrm{H}_{2}$ vibrational and rotational cooling rates.

*Now at NASA/Marshall Space Flight Center, Huntsville, AL 35812, U.S.A.
In this paper, we will provide a more concise description of these cooling rate calculations. Results will be presented for a wide range of electron and neutral temperatures. The cooling rates will be given in terms of analytical formulae as well as graphically in order to make their application to ionospheric calculations easier.

\section{VIBRATIONAL COOLING RATE}

Both excitation and de-excitation cross-sections are necessary in order to obtain the electron energy loss function required for the vibrational cooling rate calculation. Cross-sections for the $0-1$ vibrational transition $\left(\sigma_{10}\right)$ and the $0-2$ transition $\left(\sigma_{20}\right)$ have been measured by Erhardt et al. (1968). Cross-sections for de-excitation can be related to the excitation cross sections by equating the collision strengths-a consequence of time reversal symmetry (Mott and Massey, 1965). Using this procedure the de-excitation cross-sections $\sigma_{01}$ and $\sigma_{02}$ can be obtained as:

$$
\begin{gathered}
\sigma_{01}(E)=\frac{E+W}{E} \sigma_{10}(E+W) \\
\sigma_{02}(E)=\frac{E+2 W}{E} \sigma_{20}(E+W)
\end{gathered}
$$

where $E$ is energy and $W=0.54 \mathrm{eV}$ is the vibrational threshold. We make the simplifying assumption that $\mathrm{H}_{2}$ can be described as a simple harmonic oscillator, in which case the energy 
separation of all adjacent vibrational levels is $W$.

When the neutral temperature is sufficiently large so that the $V^{\prime \prime}=1$ and higher vibrational levels of $\mathrm{H}_{2}$ are populated, transitions like 1-2 or $2-1$ also need to be included. We relate the crosssections for these transitions to $\sigma_{01}, \sigma_{10}, \sigma_{02}$ and $\sigma_{20}$ by:

$$
\begin{aligned}
& \sigma_{V^{\prime \prime}+\Delta V, V^{\prime \prime}}=\sigma_{\Delta V, 0} \\
& \sigma_{V^{\prime \prime}, V+\Delta V}=\sigma_{0, \Delta V}
\end{aligned}
$$

where $\Delta V$ can equal 1 or 2 . We ignore transitions with $\Delta V=3$ or greater since the cross-sections are quite small. This can be seen by comparing the $\sigma_{01}$, $\sigma_{02}$ and $\sigma_{03}$ cross-sections measured by Erhardt $e t$ al. (1968) at electron energies where they each reach a maximum. The ratio $\sigma_{01}: \sigma_{02}: \sigma_{03}$ is about $130: 13: 1$.

Using the assumptions and cross-sections indicated above and making the further assumption that the vibrational distribution of $\mathrm{H}_{2}$ can be approximated by a Boltzmann distribution enables us to write the electron energy loss function in terms of the neutral temperature. electron energy, and the measured $\sigma_{10}$ and $\sigma_{20}$ vibrational excitation cross-sections for $\mathrm{H}_{2}$ :

$$
\begin{array}{r}
L\left(E, T_{n}\right)=W\left\{\sigma_{10}(E)+2 \sigma_{20}(E)-\exp \left(-W / k T_{n}\right)\right. \\
\times\left[\frac{E+W}{E}\right] \sigma_{10}(E+W) \\
\left.-2 \exp \left(-2 W / k T_{n}\right)\left[\frac{E+2 W}{E}\right] \sigma_{20}(E+2 W)\right\}
\end{array}
$$

where $k$ is the Boltzmann's constant and $T_{n}$ is the neutral temperature.

The vibrational cooling rate (VCRT) can then be found by integrating the loss function (3) weighted with the electron thermal velocity and a Maxwellian distribution of electrons at a temperature, $T_{e}$ (Henry and McElroy, 1969):

$$
\begin{gathered}
\operatorname{VCRT}\left(T_{e}, T_{n}\right)=2 N_{e} N_{H_{2}} \sqrt{ }\left(\frac{2}{\pi M_{e}}\right)\left(\frac{1}{k T_{e}}\right)^{3 / 2} \int_{0}^{\infty} E L \\
\left(E, T_{n}\right) \exp \left(-E / k T_{e}\right) \mathrm{d} E .
\end{gathered}
$$

After some algebraic manipulation the vibrational cooling rate can be written in the form

$$
\operatorname{VCRT}\left(T_{e}, T_{n}\right)=\frac{8.37 \times 10^{13} N_{e} N_{H_{2}} W}{T_{e}^{3 / 2}}\left\{I_{1}(1-\right.
$$

$$
\begin{gathered}
\left.\exp \left[\frac{W}{k}\left(\frac{1}{T_{e}}-\frac{1}{T_{n}}\right)\right]\right) \\
\left.+I_{2}\left(2-2 \exp \left[\frac{2 W}{k}\left(\frac{1}{T_{e}}-\frac{1}{T_{n}}\right)\right]\right)\right\}\left(\mathrm{eV} \mathrm{cm}^{-3} \mathrm{~s}^{-1}\right)
\end{gathered}
$$

where

$$
\begin{gathered}
N_{e}=\text { electron density }\left(\mathrm{cm}^{-3}\right) \\
N_{H_{2}}=\mathrm{H}_{2} \text { density }\left(\mathrm{cm}^{-3}\right)
\end{gathered}
$$

and

$$
\begin{gathered}
I_{1}=\int_{W}^{\infty} E \sigma_{10}(E) \operatorname{cxp}\left(-E / k T_{e}\right) \mathrm{d} E \\
I_{2}=\int_{2 W}^{\infty} E \sigma_{20}(E) \exp \left(-E / k T_{e}\right) \mathrm{d} E .
\end{gathered}
$$

Approximations for the integrals $I_{1}$ and $I_{2}$ were obtained by numerically integrating the measured cross-sections for $\sigma_{10}$ and $\sigma_{20}$ (Erhardt et al., 1968) and then fitting the result to a functional form:

$$
I_{i}=\exp \left(A_{i}+B_{i} / T_{e}^{1 / 2}+C_{i} / T_{e}^{3 / 2}+D_{i} / T_{e}^{2}\right)
$$

where

$$
\begin{aligned}
& A_{1}=-34.95 \\
& B_{1}=-434.0 \\
& C_{1}=-3.915 \times 10^{4} \\
& D_{1}=8.554 \times 10^{4} \quad \text { for } 100 \mathrm{~K}<T_{e}<4000 \mathrm{~K} . \\
& A_{2}=-34.85 \\
& B_{2}=-676.3 \\
& C_{2}=-1.066 \times 10^{5} \\
& D_{2}=3.573 \times 10^{5}
\end{aligned}
$$

As a check on the derived cooling rates, the calculated rate (equation 5) was compared to the results of Henry and McElroy (19769) for a neutral temperature of $300 \mathrm{~K}$. The newly derived rate is 25-50\% (Fig. 1) larger than Henry and McElroy's results. The agreement is good considering the uncertainties in the measured cross-sections and 


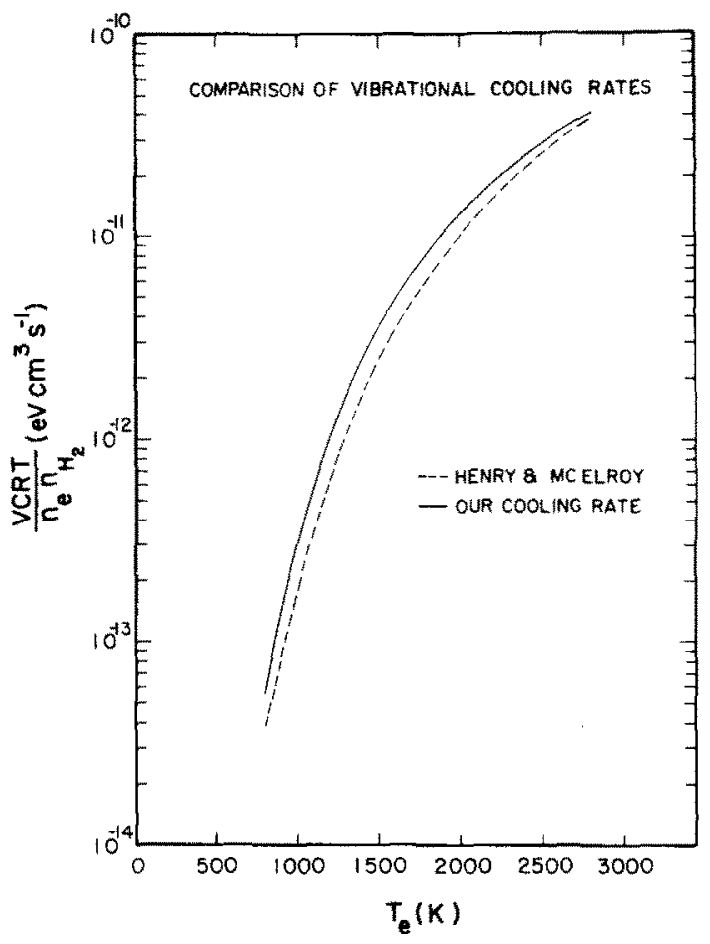

Fig. 1. Comparison of the calculated Vibrational COOLING RATE TO THAT OF HENRY AND MCELROY (1969) AS A FUNCTION OF ELECTRON TEMPFRATURE WITH $T_{n}=300 \mathrm{~K}$.

the assumptions made in the derivation. The vibrational cooling rate expression derived here (equation 5) is good not only for neutral temperatures of $300 \mathrm{~K}$ or less, like Henry and McElroy's results, but for neutral temperatures as high as several thousand degrees.

\section{DISCUSSION OF ROTATIONAL COOLING RATE}

Homonuclear molecules with non-zero nuclear spin are constrained by selection rules to be a mixture of two relatively non-interacting modifications-a symmetrical with only evennumbered rotational levels (para) and an antisymmetrical with only odd-numbered rotational levels (ortho) (Herzberg, 1950). Each population has an independent Boltzmann energy distribution with the lowest energy state for the odd population being the $J=1$ rotational state and for the even, the $J=0$ state. It is assumed there is little interaction and no thermal redistribution between the ortho and para modifications so that the cooling rate for both populations are calculated separately and then added according to their sta- tistical weights. The statistical weight of the ortho modification is three times that of the para modification and the resulting expression for the rotational cooling rate (RCRT) is:

$\mathrm{RCRT}=1 / 4$ RCRT (even) $+3 / 4 \mathrm{RCRT}$ (odd).

The derivation for both the ortho and para modifications are similar and to avoid repetition, the loss function is derived for the general case and ortho and para differences are noted when appropriate.

The rigid rotator approximation is used to obtain the transition energy:

$$
E_{J+2}-E_{J}=2 B h c(2 J+3) \text {. }
$$

As was the case for the vibrational cross-sections, time reversal arguments relate the excitation and de-excitation cross-sections:

$$
\sigma_{J^{\prime} \leftarrow J^{\prime \prime}}(E)=\frac{R_{J^{\prime} \leftarrow J^{\prime \prime}}}{R_{J^{\prime \prime} \leftarrow J^{\prime \prime}}} \frac{E+W_{J^{\prime}, J^{\prime \prime}}}{E} \sigma_{J^{\prime \prime} \leftarrow J^{\prime}}\left(E+W_{J^{\prime}, J^{\prime \prime}}\right)
$$

where the $R$ factors are the degeneracy factors taken from the work of Chang and Temkin (1969) and $W_{J^{\prime}, y^{\prime \prime}}$ is the $J^{\prime \prime} \rightarrow J^{\prime}$ threshold energy. An extension of this assumption enables us to further relate the excitation cross-section for transition $J$ to $J^{\prime}$ to the first order rotational excitation cross-sections $\sigma_{31}$ (odd) or $\sigma_{20}$ (even):

$$
\begin{aligned}
\sigma_{y^{\prime} \rightarrow J}(\mu) & = \\
& \frac{R_{J^{\prime}+J}}{R_{(31) \text { or (20) }}} \frac{\mu-\left[W_{J^{\prime}, J}-W_{(31) \text { or (20) }]}\right.}{\mu} \sigma_{(31) \text { or (20) }} \\
& \left\{\mu-\left[W_{j^{\prime}, y}-W_{(3) \text { or (20) }}\right]\right\} .
\end{aligned}
$$

Equation (11) is the simplifying assumption that enables us to separate out the higher order rotational transitions in terms of the first order transitions which have measured cross-sections and can be numerically integrated. The result is an electron energy loss function that can be defined in terms of the electron energy, the neutral temperature, and the $\sigma_{20}$ and $\sigma_{31}$ excitation crosssections. This loss function is in the form of a summation over the rotational quantum number $J$. If we integrate this loss function over a Maxwellian distribution of electron energies, the following expression for the rotational cooling rate is 
obtained:

$\operatorname{RCRT}($ odd $)=\frac{8.37 \times 10^{13} n_{e} n_{\mathrm{H}_{2}}}{T_{e}^{3 / 2}} \exp \left(\frac{2 B h c}{k T_{n}}\right) \exp \left(\frac{10 B h c}{k T_{e}}\right)$

$$
\frac{S B h c}{Q_{\text {odd }}} I_{\text {odd }}^{\prime}\left(T_{e}\right) S U M_{\text {odd }}\left(\mathrm{eV} \mathrm{cm}^{-3} \mathrm{~s}^{-1}\right)
$$

$\operatorname{RCRT}($ even $)=\frac{8.37 \times 10^{13} n_{e} n_{H_{2}}}{T_{e}^{3 / 2}}$

$$
\exp \left(\frac{6 B h c}{k T_{\varepsilon}}\right) \frac{3 B h c}{Q_{\text {even }}} I_{\text {even }}\left(T_{e}\right) S U M_{\text {even }}
$$

where,

$$
I_{i}^{\prime}\left(T_{e}\right)=\int_{W_{i}}^{\infty} E^{\prime} \exp \left(-E^{\prime} / k T_{e}\right) \sigma_{i}\left(E^{\prime}\right) \mathrm{d} E^{\prime}
$$

$$
\begin{aligned}
& \operatorname{SUM}_{i}=\sum_{\substack{\text { add } J \\
\text { vete } J}}\left\{(J+1)(J+2) \exp \left(\frac{-B J(J+1) h c}{k T_{n}}\right)\right. \\
& \left.\left[\exp \left(\frac{-2 B h c(2 J+3)}{k T_{e}}\right)-\exp \left(\frac{-2 B h c(2 J+3)}{k T_{n}}\right)\right]\right\}
\end{aligned}
$$

$i$ is an index referring either to even states or odd states. $Q$ is the partition function for ortho or para modifications.

The integral $I_{i}^{\prime}\left(T_{e}\right)$ was numerically integrated using the $\sigma_{31}$ cross-section of Linder and Schmidt (1971) and the $\sigma_{20}$ cross-section of Crompton et al. (1969). The results were then fit to the form

$$
I_{i}^{\prime}=\exp \left(A_{i}+B_{i} / T_{e}^{1 / 2}+C_{i} / T_{e}^{3 / 2}+D_{i} / T_{e}^{2}\right)
$$

where

$$
\begin{aligned}
& A_{\text {odd }}=-36.35 \\
& B_{\text {odd }}=-279.7 \\
& C_{\text {odd }}=2.366 \times 10^{4} \\
& D_{\text {odd }}=-1.791 \times 10^{5} \\
& A_{\text {even }}=-35.41
\end{aligned}
$$

$$
\begin{aligned}
& B_{\text {even }}=-306.2 \\
& C_{\text {even }}=-4.141 \times 10^{4} \\
& D_{\text {even }}=1.265 \times 10^{5}
\end{aligned}
$$

The fit is accurate to better than $5 \%$.

The final rotational cooling expression is still quite complicated. A computer code was written to calculate the proper expression for a given $T_{e}$ and $T_{n}$. To check the result the expression was compared to the cooling rate of Henry and $\mathrm{McEl}$ roy $(1969)$ at a neutral temperature of $300 \mathrm{~K}$. The results are shown in Fig. 2. The agreement ranges from a $-45 \%$ near $T_{e}=350 \mathrm{~K}$ to a $+35 \%$ near $T_{e}=2000 \mathrm{~K}$. The agreement was considered to be quite good concidering the various approximations required and the uncertainties in the measured cross-sections at low energies near the rotational thresholds.

The rotational cooling rate is still not in a very useful form. The cooling rate is shown graphically for several neutral temperatures and as a function of $T_{\varepsilon}-T_{n}$ (Fig. 3). The variation of the cooling rate with $T_{n}$ was not very large except near the threshold, so the results for a neutral temperature

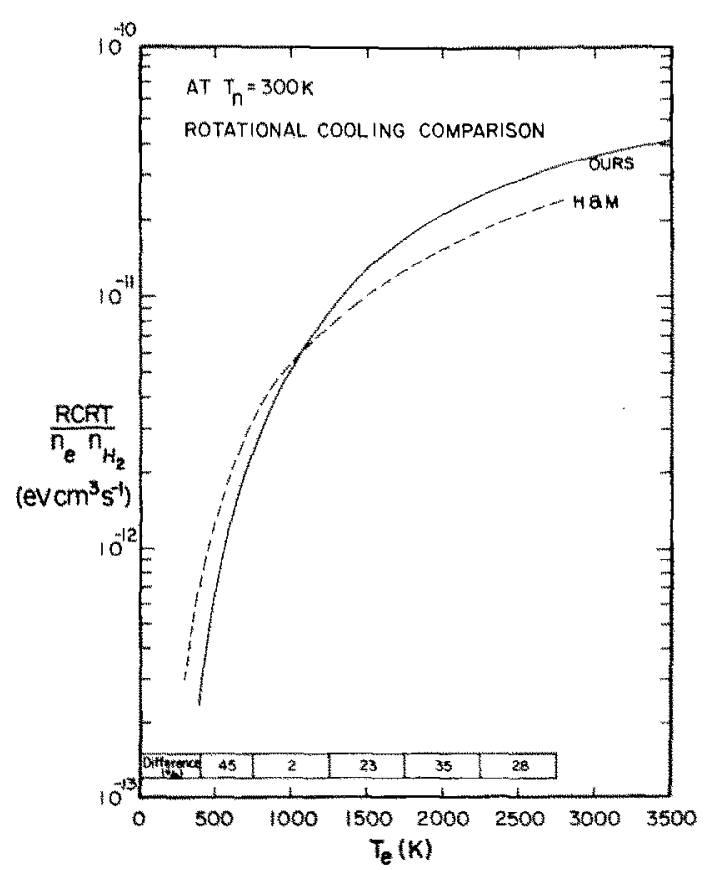

FIO. 2. COMPARISON OF THE CALCULATED ROTATIONAL COOLING RATE TO THAT OF HENRY AND MCELROY (1969) AS A FUNCTION OF ELECTRON TEMPERATURE WTH $T_{n}=300 \mathrm{~K}$. 


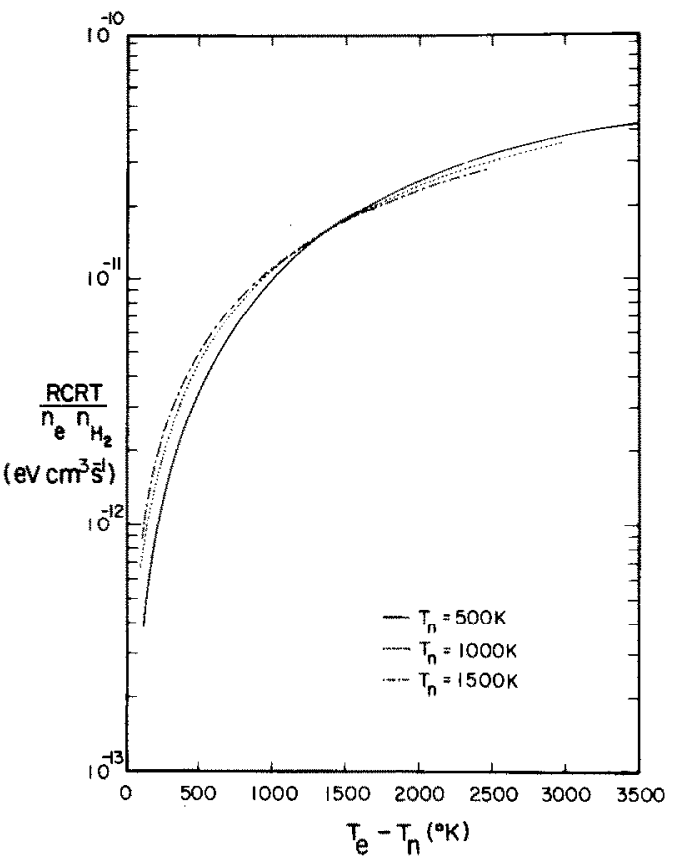

Fig. 3. The Calculated rotational CoOling Rate PLOTTED AS A FUNCTION OF THE DIFFERENCE $T_{\varepsilon}-T_{n}$ AT SEVERAL DIFFERENT $\mathrm{H}_{2}$ TEMPERATURES.

of $T_{n}=1000 \mathrm{~K}$ were fit with a functional form:

$$
\operatorname{RCRT}\left(T_{n}=1000 \mathrm{~K}\right)=A\left\{\exp \left[B\left(T_{e}-T_{n}\right)^{r}-1\right]\right\}
$$

where

$$
\begin{aligned}
& A=2.278 \times 10^{-11} \\
& B=2.093 \times 10^{-4} \\
& c=1.078 .
\end{aligned}
$$

The fit is shown in Fig. 4. Although the functional form is not accurate for a wide range of $T_{n}$, for plasma temperature calculations on Saturn and Jupiter it is adequate since the exospheric neutral temperatures for these planets are of the order of $1000 \mathrm{~K}$.

\section{SUMMARY}

In order to properly calculate the electron temperature on planets like Jupiter and Saturn where the neutral temperature is greater than $1000 \mathrm{~K}$,

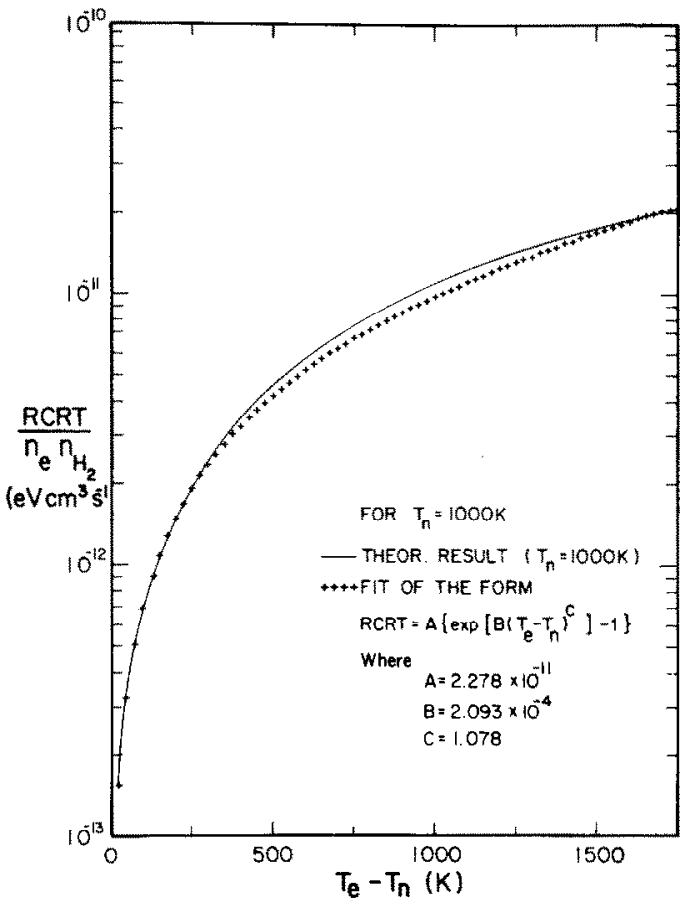

Fig. 4. THE Rotational COOLING Rate CalCUlated at a NEUTRAL TEMPERATURE OF $1000 \mathrm{~K}$ COMPARED TO THE FUNCTIONAL FIT USED IN THE ION AND ELECTRON TEM. PERATURE MODEL.

appropriate vibrational and rotational cooling rates for electrons in $\mathrm{H}_{2}$ are required. In this paper, such cooling rates were presented for a wide range of electron and neutral temperature values. Convenient analytical formulae for these cooling rates were also provided.

Acknowledgements-The authors thank R. J. W. Henry and A. F. Nagy for useful discussions. This work was supported by NASA Grants NAGW-15 and NGR 23-005015 .

\section{REFERENCES}

Atreya, S. K., Donahue, T. M., Sandel, B. R., Broadfoot, A. L. and Smith, G. R. (1979). Jovian upper atmospheric temperature measurement by the Voyager $1 \mathrm{UV}$ spectrometer, Geophys. Res. Lett. 6, 795.

Chang, E. S. and Temkin, A. (1969). Rotational excitation of diatomic molecules by electron impact. Phys. Rev. Lett. 23, 399.

Cravens, T. E. (1974). Astrophysical applications of electron energy deposition in molecular hydrogen. $\mathrm{Ph}$. D. Thesis, Harvard University.

Crompton, R. W., Gibson, D. K. and McIntosh, A. I. (1969). The cross sections for the $J=0 \rightarrow 2$ rotational excitation of $\mathrm{H}_{2}$ by slow electrons. Aust. J. Phys. 22, 715.

Erhardt, H., Langhans, L., Linder, F. and Taylor, H. S. 
(1968). Resonance scattering of slow electrons from $\mathrm{H}_{2}$ and $\mathrm{CO}$ angular distributions. Phys. Rev. 173, 222.

Fjeldbo, G., Kliore, A., Sidel, B., Sweetnam, D. and Woiceshyn, P. (1976). The Pioneer 11 radio occultation measurements of the Jovian ionosphere, in Jupiter. (Ed. T. Gehrels), The University of Arizona Press, Tucson, Arizona.

Henry, R. J. W. and McElroy, M. B. (1969). The absorption of extreme ultraviolet radiation by Jupiter's upper atmosphere, J. atmos. Sci. 26.

Herzberg, G. (1950). Molecular Spectra and Molecular Structure I. Spectra of Diatomic Molecules. Van Nostrand Reinhold. New York.

Kliore, A. J., Patel, I. R., Lindal, G. F., Sweetnam, D. N., Hotz, H. B,., Waite, J. H. Jr. and McDonough, T. R. (1980). Structure of the ionosphere and atmosphere of
Saturn from Pioneer 11 Saturn radio occultation. J. geophys. Res. 85, 5857.

Linder, F. and Schmidt, H. (1971). Rotational and vibrational excitation of $\mathrm{H}_{2}$ by slow electron impact. $Z$. Naturf. 260, 10.

Mott, N. F. and Massey, H. S. W. (1965). The Theory of Atomic Collisions. Oxford University Press, Oxford.

Nagy, A. F., Chameides, W. L., Chen, R. H. and Atreya, S. K. (1976). Electron temperatures in the Jovian ionosphere. I. geophys. Res. 81, 5567.

Prasad, S. J. and Capone, C. A. (1971). The Jovian ionosphere: composition and temperature. Icarus $\mathbf{1 5}$, 45.

Waite, J. H. Jr. (1980). The ionosphere of Saturn Ph. D. Thesis, University of Michigan. 